\title{
Personalized Low-Energy Defibrillation Through Feedback Based Resynchronization Therapy
}

\author{
Ilija Uzelac ${ }^{1}$, Flavio H Fenton ${ }^{1}$ \\ ${ }^{1}$ School of Physics - Georgia Institute of Technology, USA
}

\begin{abstract}
Aims: Defibrillation shocks may cause AV node burnout, scar formation, and pain. In this study, we present a realtime feedback-based control of ventricular fibrillation (VF) with a series of low-energy shocks using ventricular electrical activity as the feedback input.

Methods: Isolated rabbit hearts were Langendorff perfused and stained with a fluorescent $V_{m}$ dye. The ventricular activity was measured with a pair of photodiodes, and processed with a feedback controller to calculate defibrillation shock parameters in real-time. Shock timing was based on desynchronized activation of the left and right ventricles during $V F$, and the strength was proportional to the amplitude difference of the photodiode signals. Shocks were delivered with a custom-developed arbitrary waveform trans-conductance amplifier.

Results: Feedback based resynchronization therapy converts VT to MT before sinus rhythm is restored with a reduction of defibrillation energy, compared to a single biphasic shock.

Conclusions: Feedback based resynchronization therapy is based on real-time sensing of ventricular activity, while a series of low-energy shocks are delivered, reducing the risk of associated side effects.

Keywords - Low-energy defibrillation, feedback control, optical mapping.
\end{abstract}

\section{Introduction}

Sudden cardiac death is a major health issue worldwide, with approximately 350,000 deaths each year in the United States. Out of those, one third is attributed to ventricular fibrillation (VF) [1]. Once initiated, VF is selfperpetuated by re-entrant waves, and the only effective treatment method is to deliver an electric shock.

Single electric shock is a widely accepted method for resynchronization therapy, with no significant change since inception in the mid-'60s. Although defibrillation waveforms were optimized over time, leading to biphasic waveforms, the waveforms are not optimized to a current heart condition and complexity of VF. Many manufactur- ers and research groups usually use energy dosages as a comparison tool to evaluate the efficacy of different waveforms. However, a comparison of energy dosages is not necessarily a valid comparison tool. As with two drugs of different molecular formulation aimed to treat the same condition, the dosage of each drug is not necessarily the same. Therefore, comparing the therapeutic strength measured in Joules between two defibrillation waveforms can be misleading to determine which one is better. Among these reasons, the American Heart Association advocates that it is the electrical current that defibrillates and not the amount of energy [2]. Also among published literature, the current is considered as a better predictor for a successful resynchronization strategy $[3,4]$.

Defibrillation shocks side effects include electroporation with a possibility of scar formation, AV node burnout risk, and pain experienced in conscious patients $[5,6]$. Scar formation facilitates VF re-occurrence providing further anchoring point for a reentrant waves, and the formation of a spiral waves [7]. Single shock defibrillation needs to excite enough myocardial tissue at once, liking importance of a single shock timing with the actual VF dynamics [8-10]. In this study, we show that the delivery of a series of lowenergy shocks using real-time feedback to determine the shock's timing, strength and duration can successfully terminates VF.

\section{Methods}

Optical mapping experiments were performed on isolated New Zealand White rabbit's hearts in Langerdorff perfusion. All experimental procedures were approved by the Office and Research Integrity Assurance of Georgia Tech under IACUC protocol A15034. The hearts were stained with Di-4-ANBDQPQ transmembrane voltage $\mathrm{V}_{m}$ dye, while motion contraction was suppressed with Blebbistatin at the perfusate concentration of 1.8 $\mu \mathrm{M}$. A PLUMBUS (Pulsed LUMinos Bimodal Uniform Source), provided by Aleksa Tech, was used as an LED illumination system, powering red LEDs with a center wavelength of $660 \mathrm{~nm}$, Figure 1.

Due to the relatively small size of rabbit hearts, it is 


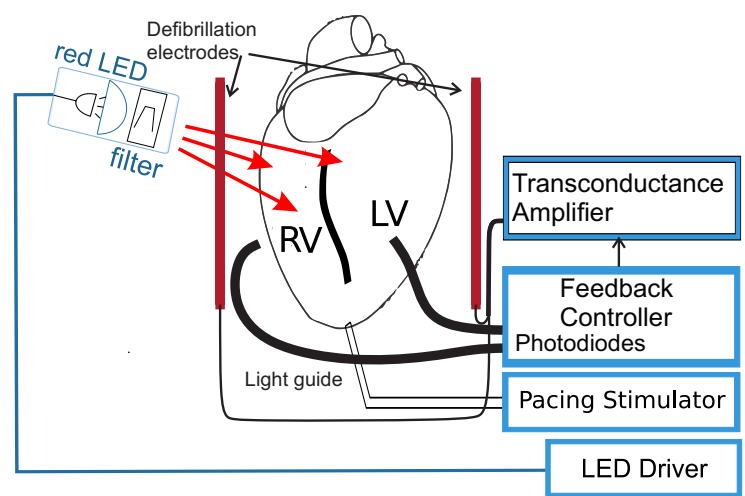

Figure 1. Experimental setup. The anterior surface of the heart was illuminated and two light guides were used to gather emitted fluorescence light from the left (LV) and right ventricles (RV) to the feedback controller consisting of a pair of photodiodes, and an embedded system to process the input data. The feedback controller computes a control signal (defibrillation shock) to actuate the transconductance amplifier able to deliver an arbitrary waveform current defibrillation shock through a pair of defibrillation electrode plates placed at opposite sides of the heart.

possible to monitor the ventricular activity across large areas by placing two light guides facing the anterior surface of the LV and RV respectively to gather emitted fluorescence to the photodiodes. The feedback controller based on an ARM class 32-bit micro-controller processes the input data from photodiodes sampled at $2 \mathrm{kHz}$. The difference between the two input signals represents a measure of desynchronized activation between the LV and RV. Any non-zero difference exceeding the experimentally defined threshold served as a trigger signal for early detection of the onset of arrhythmia, Figure 2. The threshold is experimentally determined during sinus rhythm, by placing the light guides at different positions until the difference signal is minimized.

During sustained VF, the timing of the electric shocks is based on the asynchronous activation times from the monitored ventricular areas, coinciding with the periods around the dominant frequency during VF. The feedback controller counts the time from the last shock, and if the time exceeds a predefined time interval longer than the period associated with the dominant frequency it delivers another shock. The predefined interval can be automatically calculated from the VF signal spectra. The amplitude is calculated in the real-time feedback loop every $0.5 \mathrm{~ms}$, based on the difference between the two photodiode signals. The feedback controller calculates optimized defibrillation shock waveform, and a custom-built transconductance amplifier (T-amp), provided by Aleksa Tech) delivers an electrical shock in the form of the electrical cur-

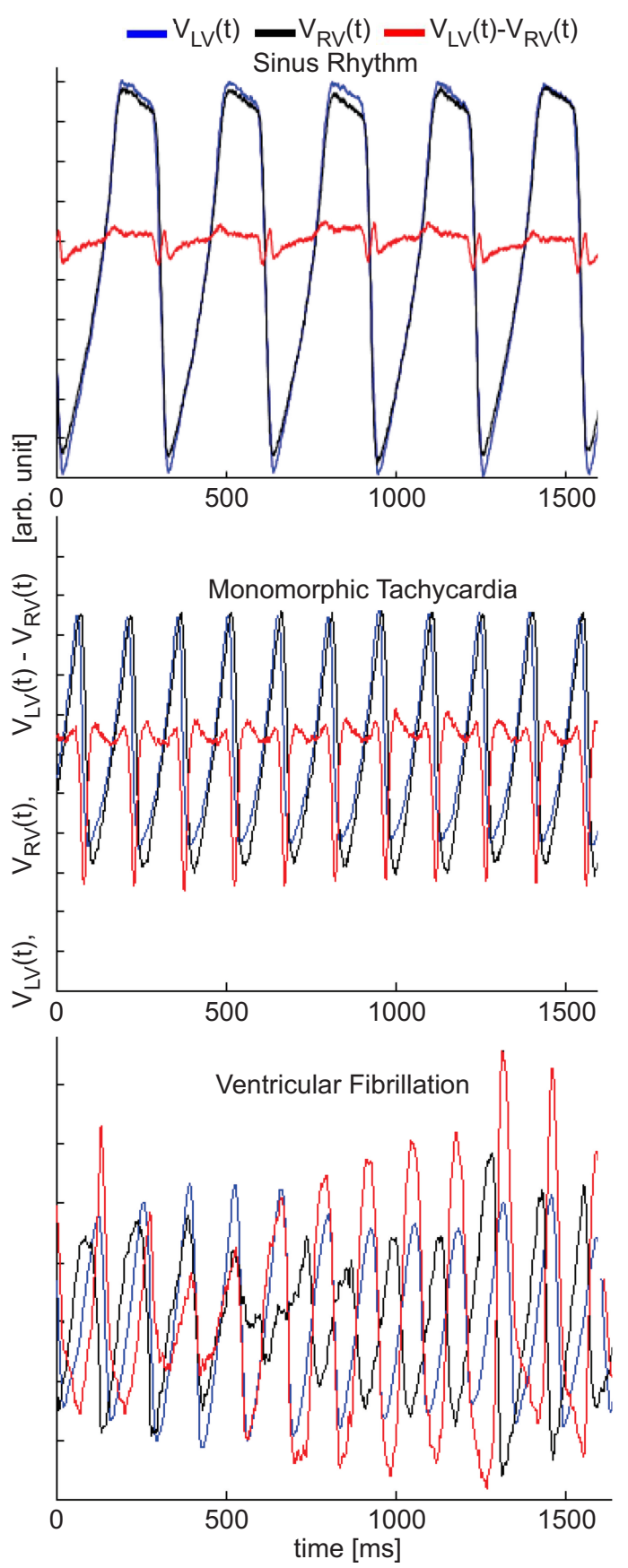

Figure 2. Fluorescence signals $\mathrm{V}_{L V}(\mathrm{t})$ and $\mathrm{V}_{R V}(\mathrm{t})$ representing action potentials (inverted) from the two anterior sites at the ventricles. During sinus rhythm both signals are in phase, and of the same amplitude, resulting in a difference signal of low-amplitude (red trace). During monomorphic tachycardia (MT), both signals are of the same amplitude, but of different activation times (out of phase) resulting in a non-zero difference signal exceeding both $\mathrm{V}_{L V}(\mathrm{t})$ and $\mathrm{V}_{R V}(\mathrm{t})$ amplitudes. During VF, both signals are out of phase and of different amplitudes resulting in a non-zero difference signal of significantly larger amplitude. 
rent, proportional to the calculated waveforms. Over the time interval of few seconds VF is converted to MT and ventricular activity is synchronized at the frequency lower than the dominant frequency during VF.

\section{Results}

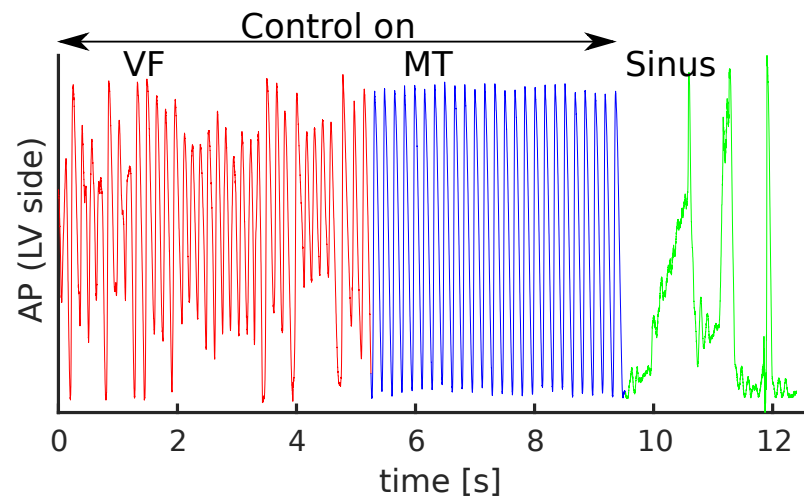

Figure 3. Optical AP trace representing electrical activity from the imaged anterior area of the LV. During VF, the feedback controller was turned on and over the period of 5 $\mathrm{s}$, the ventricular electrical activity was synchronized with the self-determined period of low-energy shocks, resulting in the conversion of VF to MT. Upon synchronization, the external controller keeps stimulating the ventricles with the period of the predefined time interval, as during MT both ventricles are synchronized resulting in the subthreshold difference signal. The controller keeps the ventricles activating in synchronicity and prevents the development of VF. Sinus resumed after the control was turned off
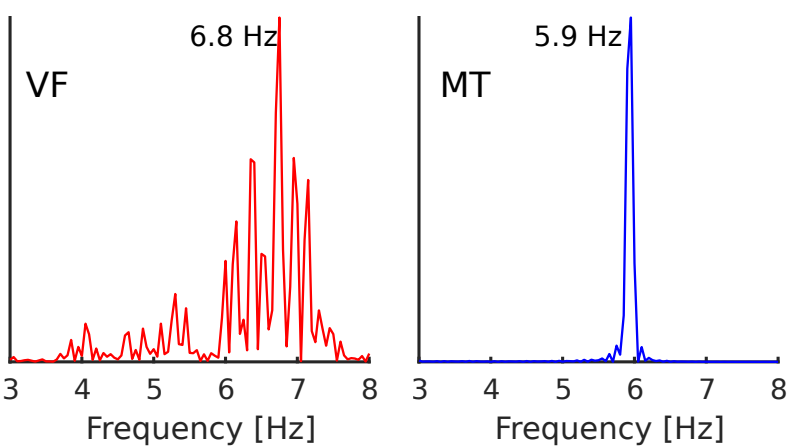

Figure 4. Frequency spectra of the optical AP signal. During $\mathrm{VF}$, the frequency spectrum has a broad peak around the dominant frequency of $6.8 \mathrm{~Hz}$ which indicates complex VF driven with the multiple spiral waves, and dynamically induced heterogeneities during tissue repolarization and depolarization. Upon successful resynchronization, a sharp peak indicates the presence of organized ventricular activity externally driven with the feedback controller.

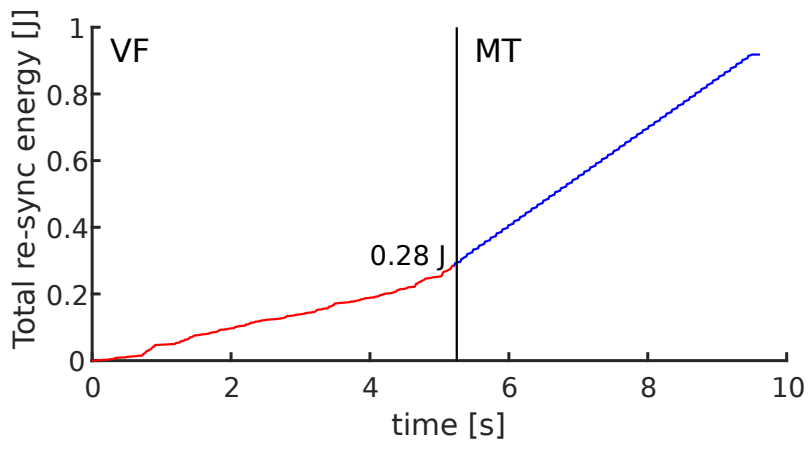

Figure 5. The total energy delivered to the heart for time duration while the control was turned on. The energy is delivered through a series of low-energy shocks achieving conversion from VF to MT in the time interval of approximately $5 \mathrm{~s}$, while the total energy was less than $0.3 \mathrm{~J}$.

In the feedback control experiments, the optical action potential (AP) signals representing ventricular electrical activity from the LV and RV areas were acquired and processed in real-time. The aim was to study resynchronization strategies to achieve the optimization for the optimal placement of the light-guides, timing of low-energy shock while limiting the maximal strength of each shock. VF was induced via burst pacing protocol and sustained VF was determined if not self-terminate within one minute. During sustained VF, the control was turned on, Figure 3, and series of a low-energy shocks were delivered to locally perturb the ventricular activity at the sites next to the defibrillation electrodes. The period between low-energy shocks is automatically calculated from the input signals of the two photodiodes via real-time feedback loop[11,12]. In most experiments, the time needed to reach the synchronous activity of the LV and RV was 4 - 6 seconds when VF was converted to MT, Figure 3. Upon achieving conversion to MT, the external control was kept on to maintain the MT and to prevent the development of VF. It is important to emphasize that the control could be terminated at any instance during a stable MT episode when sinus rhythm resumes.

The frequency spectra of the LV activity is shown in Figure 4. A series of low-energy shocks synchronizes first with the dominant frequency of VF suppressing all the frequencies other than the dominant frequency, leading to the re-synchronized activity of the LV and RV. Upon resynchronization, the shocks are continuously delivered at a frequency smaller than the VF dominant frequency. The sharp dominant frequency peak in the right panel indicates the reduction of VF to MT, when the external controller acts a pacemaker but also prevents MT degradation into VF.

The total delivered energy during VF conversion to MT in most experiments was less than $0.5 \mathrm{~J}$ and often under 
$0.3 \mathrm{~J}$ as shown in Figure 5, for the total duration of around $5 \mathrm{~s}$. During the conversion time of $5 \mathrm{~s}$, approximately 35 low-energy shocks were delivered or approximately only 8 $\mathrm{mJ}$ per single shock.

\section{Discussion}

In the presented feedback-based resynchronization therapy studies, it is important to minimize the phase difference between the LV and RV during normal sinus rhythm. The phase difference between the two photodiode signals depends on the location of the placement of the light guides next to the ventricles, and the distance from the heart. In a series of resynchronization experiments, we observed a correlation between the necessary conversion time from VF to MT, and the complexity of VF. The conversion time of a few seconds may seem unacceptably long. However, a single-shock based defibrillation delivers all the energy in less than $20 \mathrm{~ms}$. In contrast, our defibrillation strategy delivers less overall energy over two orders of magnitude longer time interval. The determined energy threshold for a single $10 \mathrm{~ms}$ biphasic shock in the experimental setup was around $0.4 \mathrm{~J}$, consistent with a previously published study in isolated rabbit heart preparations using external defibrillation pads [13].

\section{Conclusions}

The main purpose of this study was to investigate feedback based resynchronization strategies with low defibrillation energies. Similarly to LEAP $[8,14,15]$ defibrillation method, the proposed feedback based defibrillation works through gradual synchronization of the ventricular activity to the same dominant frequency during VF with each additional shock. Direct comparison of two defibrillation methods should not be only based on the amount of defibrillation energy needed, but also on the instantaneous power or the time interval needed. With the presented method, the total energy applied is not only lower or comparable to the energy of a single biphasic shock but also delivered across two orders of magnitude longer time interval. The pain and damage can be minimized with the delivery of defibrillation energy over a longer time interval using alternative approaches of the same efficiency as the single shock based defibrillation. Additionally, as the timing, strength, and frequency of the sequence of low-energy shocks are based on the local VF dynamics, the resynchronization method presented here, is "personalized" or specific to the actual VF dynamics independent of mechanism.

\section{Acknowledgments}

We acknowledge support from the following grants: NIH 1R01HL143450-01, and NSF 1762553

\section{References}

[1] Almahameed ST, Kaufman ES. Idiopathic ventricular fibrillation: Diagnosis, ablation of triggers, gaps in knowledge, and future directions. The Journal of Innovations in Cardiac Rhythm Management 2020;11(6):4135.

[2] Association AH, et al. American heart association guidelines for cardiopulmonary resuscitation and emergency cardiovascular care. part 7.2: Management of cardiac arrest. Circulation 2005;112(Suppl 24):78-83.

[3] Uzelac I, Holcomb M, Reiserer RS, Fenton FH, Wikswo JP. High-power current source with real-time arbitrary waveforms for in vivo and in vitro studies of defibrillation. In Computing in Cardiology 2013. IEEE, 2013; 667-670.

[4] Ristagno G, Yu T, Quan W, Freeman G, Li Y. Current is better than energy as predictor of success for biphasic defibrillatory shocks in a porcine model of ventricular fibrillation. Resuscitation 2013;84(5):678-683.

[5] Walcott GP, Killingsworth CR, Ideker RE. Do clinically relevant transthoracic defibrillation energies cause myocardial damage and dysfunction? Resuscitation 2003;59(1):59-70.

[6] Gerstein NS, McLean AR, Stecker EC, Schulman PM. External defibrillator damage associated with attempted synchronized dual-dose cardioversion. Annals of emergency medicine 2018;71(1):109-112.

[7] Jalife J, Gray RA, Morley GE, Davidenko JM. Selforganization and the dynamical nature of ventricular fibrillation. Chaos An Interdisciplinary Journal of Nonlinear Science 1998;8(1):79-93.

[8] Ji YC, et al. Synchronization as a mechanism for low-energy anti-fibrillation pacing. Heart rhythm 2017; 14(8):1254-1262.

[9] Marn-Pernat A, Weil MH, Tang W, Pernat A, Bisera J. Optimizing timing of ventricular defibrillation. Critical care medicine 2001;29(12):2360-2365.

[10] Patwardhan AR, Leonelli FM. Multidirectional ecg coherent optimal timing of defibrillation shocks, April 211998. US Patent 5,741,304.

[11] Rappel WJ, Fenton F, Karma A. Spatiotemporal control of wave instabilities in cardiac tissue. Physical review letters 1999;83(2):456.

[12] Garzón A, Grigoriev RO, Fenton FH. Continuous-time control of alternans in long purkinje fibers. Chaos An Interdisciplinary Journal of Nonlinear Science 2014;24(3):033124.

[13] Holley LK, McCulloch RM. Comparison of biphasic and monophasic defibrillation waveforms in an isolated rabbit heart preparation. Cardiovascular research 1991; 25(12):979-983.

[14] Fenton F, et al. Termination of atrial fibrillation using pulsed low-energy far-field stimulation. Circulation 2009; 120(6):467.

[15] Luther S, Fenton FH, Kornreich BG, Squires A, Bittihn P, Hornung D, Zabel M, Flanders J, Gladuli A, Campoy L, et al. Low-energy control of electrical turbulence in the heart. Nature 2011;475(7355):235-239.

Address for correspondence:

Ilija Uzelac, uzelaci@gmail.com 